\title{
Test results of the infrared single-mode fiber for the DARWIN mission
}

\author{
Lun K. Cheng ${ }^{* a}$, Anne-Jans Faber ${ }^{\mathrm{a}}$, Wim Gielesen ${ }^{\mathrm{a}}$, Catherine Boussard-Plédel ${ }^{\mathrm{b}}$, \\ Patrick Houizot $^{b}$, Jacques Lucas ${ }^{b}$, João Pereira Do Carmo ${ }^{c}$ \\ ${ }^{\mathrm{a}}$ TNO Science and Industry, P.O. Box 155, 2600 AD Delft, The Netherlands; \\ ${ }^{\mathrm{b}}$ University of Rennes, Glass and Ceramic Laboratory, Campus of Beaulieu, 35042 Rennes, Frans; \\ ${ }^{c}$ European Space Agency, P.O. Box 299, 2200 AG Noordwijk ZH, The Netherlands
}

\begin{abstract}
Nulling interferometry is the baseline technique for the DARWIN planet finding mission of the European Space Agency. Using this technique it will be possible to cancel, by destructive interference, the light from the bright star and look directly at its surrounding planets and eventually discover life on them. To achieve this goal wavefront errors need to be reduced to a very high degree in order to achieve the required nulling quality. Such a high wavefront quality can only be achieved with adequate wavefront filtering measures. Single mode fibers in general have excellent mode filtering capabilities, but they were not recently available for the broad infrared wavelength region of Darwin (4-20 um). Within an ESA technology development project, TNO has designed and tested an infrared single mode fiber based on chalcogenide glasses that has been manufactured by the University of Rennes. Several tests are carried out to characterize the materials used and the IR single mode fiber. Far field intensity distribution measurement at 10.6 um reveals the single mode operation of the manufactured fiber. Influence of coating, length, light coupling and bending of the fiber are also investigated.
\end{abstract}

Keywords: interferometer, interferometry, astrometry, fiber

\section{INTRODUCTION}

One of DARWIN's two main objectives is to find other terrestrial planets orbiting around nearby stars. This can be done by performing a simple spectroscopic analysis of their atmosphere. This spectroscopic analysis could show that some chemical components usually associated with life are present in some exo-planets and therefore there is a possibility for extra-terrestrial life. A vital function of DARWIN is the so-called "nulling" operation [1] (see Figure 1).

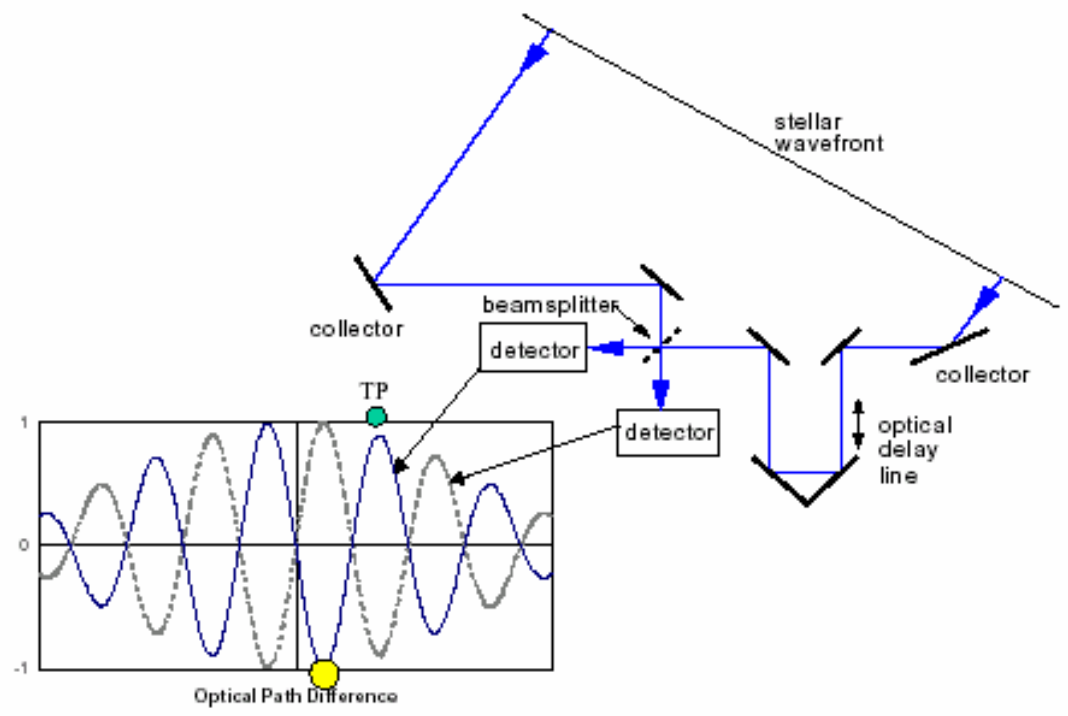

Figure 1 Basic concept of nulling interferometer.

\footnotetext{
*lun.cheng@tno.nl
} 
Even at infra-red wavelengths the flux emitted by the star is extremely large compared to the flux emitted by the planet. Without nulling, the star signal would completely mask the planet signal. The principle of nulling is based on adding a phase difference of $\pi$ radians in one of the two beams before recombining them in the focal plane so that they interfere destructively at zero optical path difference (Figure 1). The signal from a point like star on the optical axis of the constellation is thus theoretically null in the focal plane. The planet signal is not nulled because the planet is slightly off axis so that the phase between the various beams is different from that of the star. This trick reduces the contrast between star and planet and enables the detection of the planet. The challenge of nulling is making the null sufficiently deep.

The performance of the nulling is limited by a number of parameters. The star is not a perfect point so that nulling is not perfect. To decrease the sensitivity to this parameter, a specific configuration has been chosen for DARWIN with six orbiting telescopes around a central hub and a dedicated scheme for the introduction of the phase shifts on the various beams before their recombination. Furthermore, the following requirements have also to be met to achieve a sufficient nulling:

Exact phase shift difference between the beams ( $\pi$ radians for the configuration shown in Figure 1).

The phase between the beams must be extremely stable to ensure a good nulling performance during the exposure which can be up to several tens of hours.

- Matching of polarization of the beams.

- The amplitude of the beams must be the same as well as the repartition of this amplitude on the pupil so that all flux from the telescopes fully interferes.

- Nearly perfect wavefront of the beams.

The standard spatial filtering with the pinhole is not suitable for nulling because it acts as a low frequency filter with a fixed dimension. Therefore the preferable concept for nulling wavefront filtering is a single mode waveguide. For the application of the single mode waveguide as wavefront filter in the Darwin, the waveguide has to meet the operational conditions in the space like the operational temperature and the radiation susceptibility. Furthermore, a minimal insertion loss is desirable. The technical requirements for the single mode waveguide are summarized in the table below.

Table 1 Technical requirements for the single mode waveguide for wavefront filtering.

\begin{tabular}{|c|c|}
\hline Parameter & Value \\
\hline Operational wavelength range & $4-20 \mu \mathrm{m}$ \\
\hline Higher order mode suppression ratio & $10^{-6}$ \\
\hline Total insertion loss goal & $<1.5 \mathrm{~dB}$ (about 30\%) \\
\hline Operational temperature & 40 Kelvin \\
\hline Radiation susceptibility & Not susceptible to radiation levels $<10^{4}$ Rads \\
\hline Fiber manufacturing process reproducibility & Should enable $5 \%$ or better parameter realisation \\
\hline
\end{tabular}

\subsection{Suitable single mode waveguide}

A survey on single mode optical waveguide structures for wavefront filtering has been performed, including the following techniques:

- Conventional single mode optical fiber

- Integrated Optical (IO) waveguide

- Conventional hollow fiber

- Photonic Crystal Fibers (PCF)

- Fibers based on Photonic Band Gap (PBG)

Based on the comparison between the various waveguide technologies, the conventional single mode optical fiber is regarded to be the most suitable technology for meeting all essential requirements for wide wavelength range wavefront filtering for Darwin. The optical fiber is a mature technology and the design can be modelled using different standard commercial software packages. This is of importance for the development of a single mode fiber for a large wavelength range. 


\section{IR MATERIAL FOR THE DARWIN SINGLE MODE FIBER}

Currently available and potential new IR transmitting materials, suitable for the DARWIN wavelength range, have been identified and the feasibility to manufacture single mode fibers/waveguides using this materials has been investigated. Based on an extensive review, the following 2 material types are identified to be the best candidates, in order of preference:

- Chalcogenide glasses

- Crystalline silver halides

As far as known, a single mode, single-crystal halide fiber has not been demonstrated yet. The most stable glasses with transmission up to about $15 \mu \mathrm{m}$ are chalcogenide glasses. Furthermore, the technological possibilities for making single mode fibers from chalcogenide glasses are more promising and favourable compared to potential fiber technologies for crystalline halides. The chalcogenide glass used in this project is based on the Te-As-Se (TAS) composition.

Characterization of bulk TAS samples, having different Te-As-Se compositions, has been performed to determine the optical properties (transmission, refractive index, dispersion). This information is required for the fine tuning of the final waveguide design prior to the actual manufacturing of fibers. The extinction coefficients and refractive index as a function of the wavelength have been determined for several samples of 3 different TAS compositions (Figure 2) in order to define the required composition for the core and cladding of the single mode fiber .

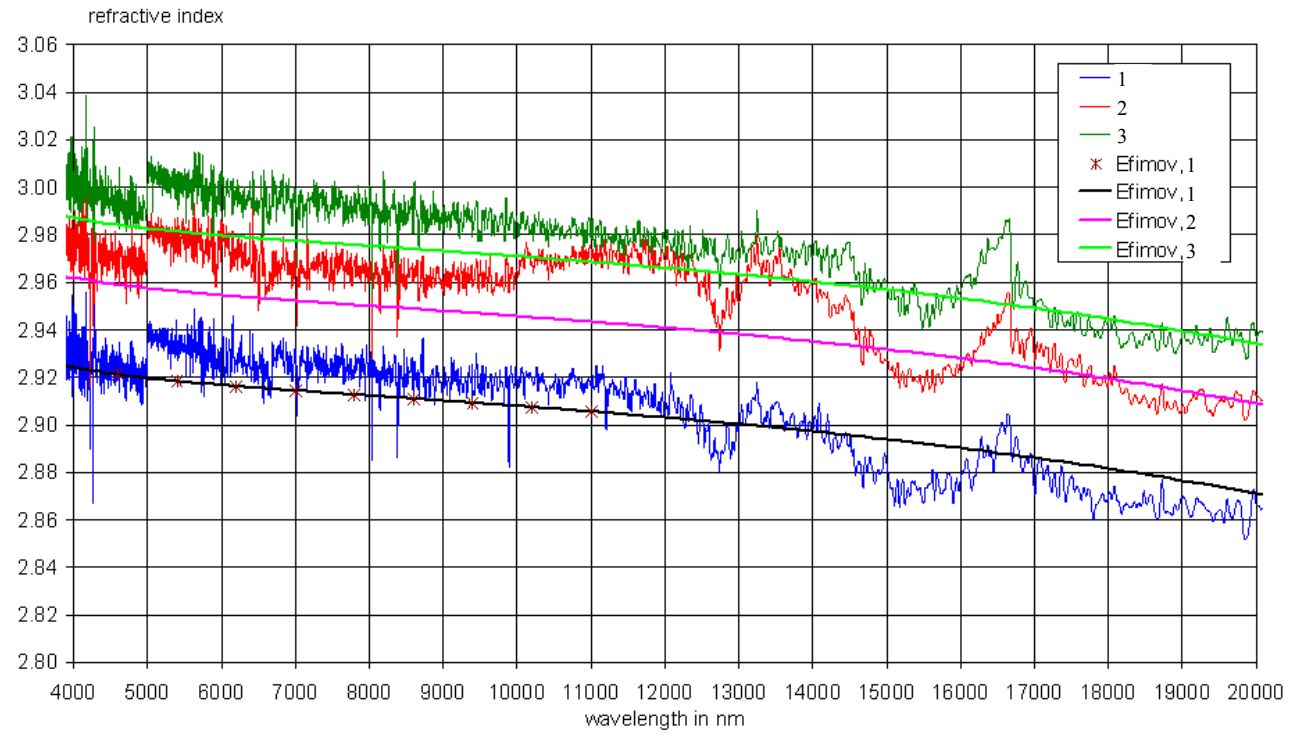

Figure 2 Refractive index result as function of wavelength

\section{DESIGN AND MAUFACTURING OF THE SINGLE MODE FIBER}

For a conventional step index single mode fiber, the coupling efficiency will drop dramatically with longer wavelength because of the exponential increase of the mode field diameter (MFD) at longer wavelengths while the Airy disc diameter of the input field is proportional to the wavelength. This prevents broadband operation of a single mode fiber for wavefront filtering. Hence, splitting the entire wavelength range into several bands is the most favourable solution. Therefore, a dual bands system consisting of two different single mode fibers for each wavelength band has been proposed.

\subsection{Design of the IR fiber}

Using the refractive index and dispersion data achieved on bulk sample testing, a final design fine tuning resulted in the following main parameters for a dual band configuration: 
Table 2 Design parameters of the dual band configuration.

\begin{tabular}{|l|c|c|c|}
\hline & Short wavelength & $\begin{array}{c}\text { Long wavelength } \\
\text { Configuration A }\end{array}$ & $\begin{array}{c}\text { Long wavelength } \\
\text { Configuration B }\end{array}$ \\
\hline Wavelength range $(\mu \mathrm{m})$ & $4-9$ & $9-20$ & $9-20$ \\
\hline Cut off $(\mu \mathrm{m})$ & 3.7 & 8.5 & 8.5 \\
\hline Core radius $(\mu \mathrm{m})$ & 11 & 26 & 15 \\
\hline Cladding thickness $(\mu \mathrm{m})$ & 250 & 600 & 350 \\
\hline n core & 2.927 & 2.913 & 2.918 \\
\hline n cladding & 2.924 & 2.910 & 2.910 \\
\hline
\end{tabular}

The manufacturing of a chalcogenide glass fiber is a highly elaborate, time consuming activity. For the first single mode fiber, the short wavelength configuration was selected because it represents the worst-case configuration for manufacturing: smallest core-radius in combination with smallest difference in refractive index between core and cladding. The manufacturing and test results of the Short Wavelength fiber is presented below.

\subsection{Manufacturing of the sort wavelength range TAS IR fibers}

\section{$\underline{T A S 1}$}

Based on the design fine tuning, implementing the refractive index and dispersion results achieved on TAS bulk sample testing, a conventional step index TAS fiber configuration for the short wavelength range $(4-9 \mu \mathrm{m})$ was specified by TNO for manufacturing the first TAS fiber (TAS-1). The University of Rennes manufactured the TAS-1 fiber using their patented novel manufacturing technique $[2,3]$ designated as Internal Rotational Casting Method (IRCM).

\section{$\underline{T A S-X}$}

Because the IRCM technique proved to be rather complex and time consuming, a second technique was explored, designated as Fiber in Tube Vacuum Method (FTVM) to manufacture the next short wavelength range TAS fiber. The fiber samples manufactured using this method is denoted as TAS-X.

\section{$\underline{T A S-3}$}

At a later stage the FTVM is improved and the new method is named the Rod in Tube Vacuum Method (RTVM). This method is used for the TAS-3 fiber.

The manufacturing methods will be described in a separate paper. Furthermore, Gallium coating will be applied to the fibers to suppress cladding modes.

\section{TEST RESULTS OF THE SHORT WAVELENGTH RANGE SINGLE MODE FIBER}

The following tests have been carried out to characterise the quality and performance of TAS fibers:

- Visual inspection

- Far Field Intensity (FFI) distribution measurement procedures and set-ups, both at $4.8 \mu \mathrm{m}$ (tuneable lead-salt laser $)$ and at $10.6 \mu \mathrm{m}\left(\mathrm{CO}_{2}\right.$-laser $)$

- Attenuation measurement at $10.6 \mu \mathrm{m}$

- Determination of the cut-off wavelength (Mode Field Diameter (MFD) measurement)

- Determination of the presence of higher order modes (Intermodal Interference Measurement (IIM))

\subsection{Visual inspection}

Visual inspection proved that the TAS fibers have a very smooth cross section. The interface between core and cladding of the regular TAS-fibers cannot be observed either by conventional microscope or SEM due to the small difference in refractive index between core and cladding (Figure 3a). To verify the core geometry of fibers manufactured by the various manufacturing methods, high-contrast (HC) fibers with a significantly high difference in the core and cladding refractive index are made. Only for the high-contrast fibers the geometry of the core can be visualized. This demonstrates circular core geometry of a fiber manufactured by the RTVM method (Figure 3b). 


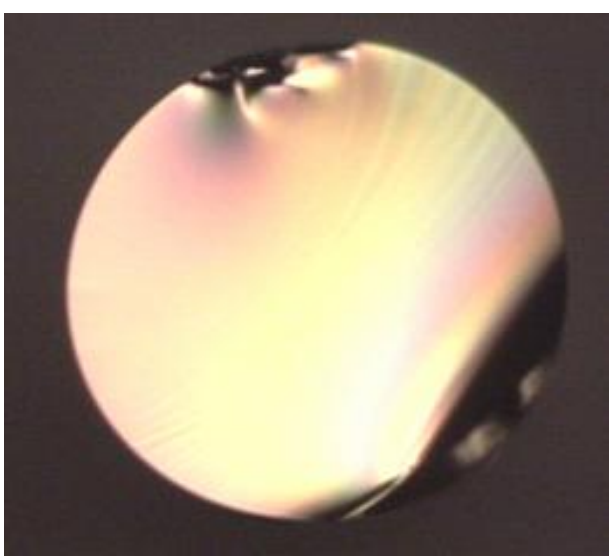

(a)

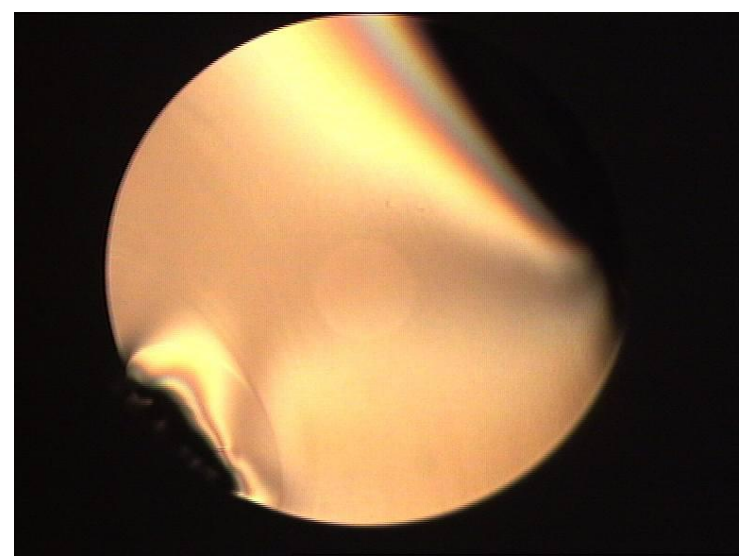

(b)

Figure 3 Cross-sections of TAS fibers under microscope: a) TAS-1 (smooth surface, no interface core-cladding visible); b) HC-TAS2 (cylindrical core).

\subsection{Far-field intersity distribution measurement at $\mathbf{1 0 . 6} \mu \mathrm{m}$}

A set-up was developed at TNO to measure 2D Far Field Intensity distribution at $10.6 \mu \mathrm{m}$ using a $\mathrm{CO}_{2}$-laser and a 2D IR camera (Figure 4). A pinhole in front of the fiber ensures that the laser light is mainly coupled into the core of the fiber.

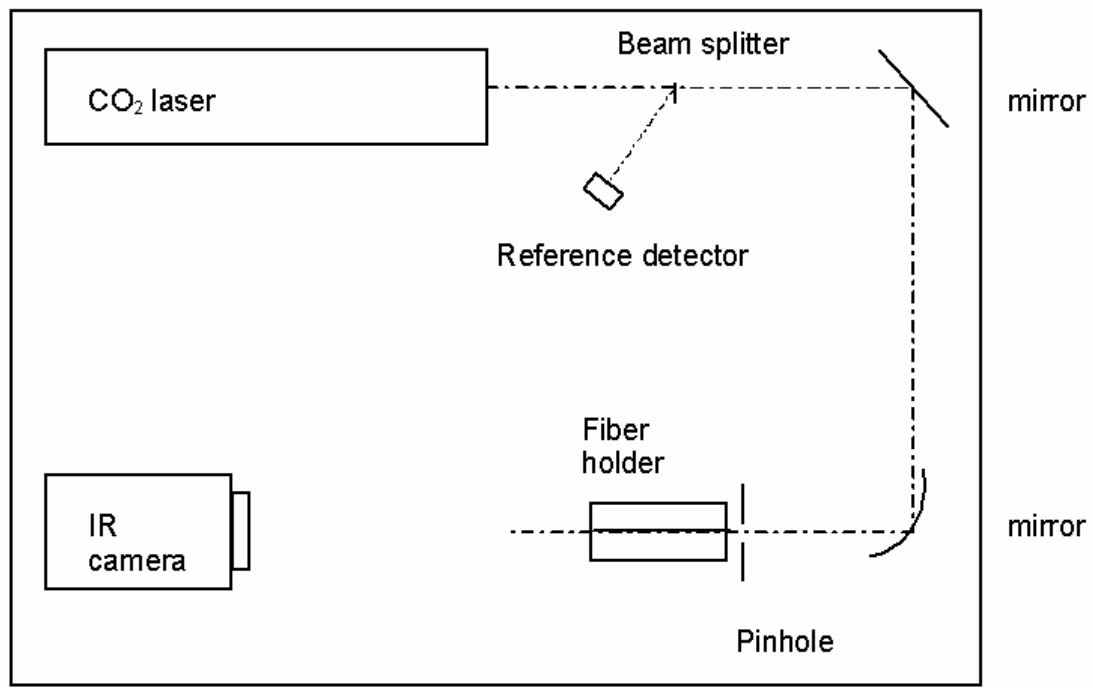

Figure 4 Schematic configuration of $\mathrm{CO}_{2}$-laser FFI distribution set-up

With this set-up, a number of tests on samples of TAS-1 was conducted to show the influence of the following parameters on the performance of a TAS-fiber:

- Presence and quality of absorbing Gallium coating for stripping of cladding modes

- Length of fiber necessary to suppress higher order modes in order to achieve single mode operation

- Mismatching of light incoupling

\section{Effect of Gallium coating}

The first tests with a $30 \mathrm{~cm}$ long TAS-1 fiber without Ga coating shows the presence of many cladding modes. No main peak of the fundamental mode can be discriminated. Learning from these tests, a TAS-1 fiber sample was prepared, having a length of $8 \mathrm{~cm}$, properly coated with Gallium over it's entire length. After optimising the light coupling by accurate alignment, the Far Field Intensity distribution was measured showing clearly a main peak (Figure 5). 


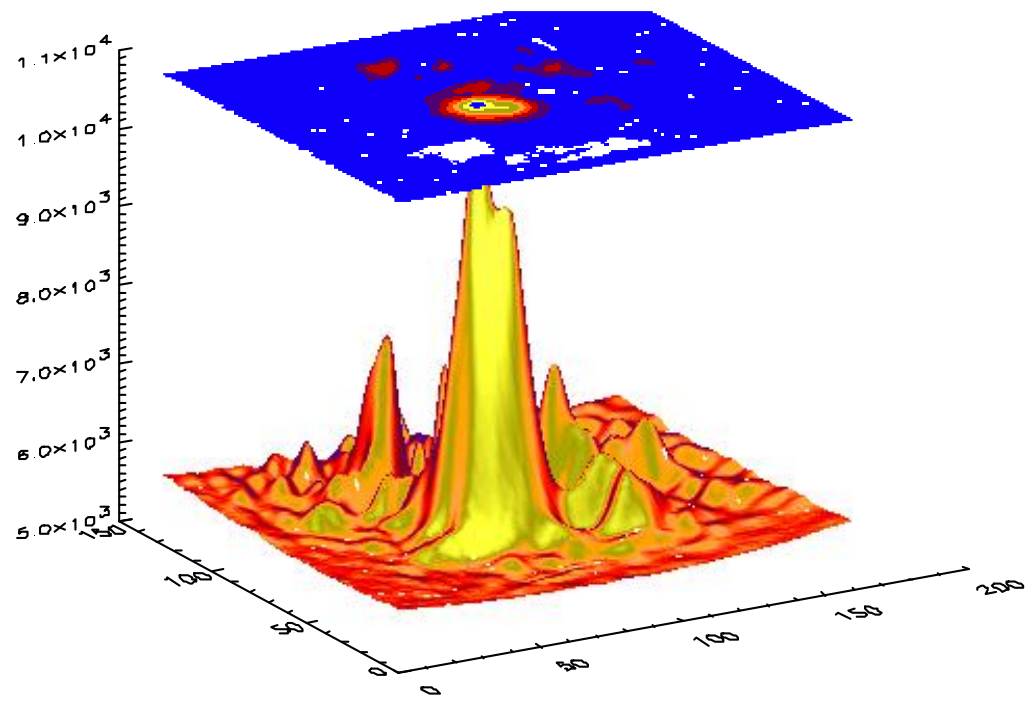

Figure 5 FFI of sample TAS-1D (8cm, Gallium coated).

\section{Effect of fiber length}

Gallium coating is demonstrated to be able to strip cladding modes (Figure 5). The TAS-1 fiber with a length of $8 \mathrm{~cm}$ and Gallium coating is found to still contain higher order and/or cladding modes. Therefore a longer fiber of $23 \mathrm{~cm}$ is tested. After optimizing the light coupling, the far-field intensity distribution is measured. Increasing the length of the TAS- 1 from 8 to $23 \mathrm{~cm}$ shows the suppression of the cladding modes, the remaining peak with a Gaussian intensity distribution indicates single mode operation of the fiber (Figure 6). However, it can be clearly observed that the tested TAS-1 fiber doesn't have a circular output intensity distribution but is more an ellipse. This can be caused by either mechanical stress introduced in the fiber during the fabrication process.
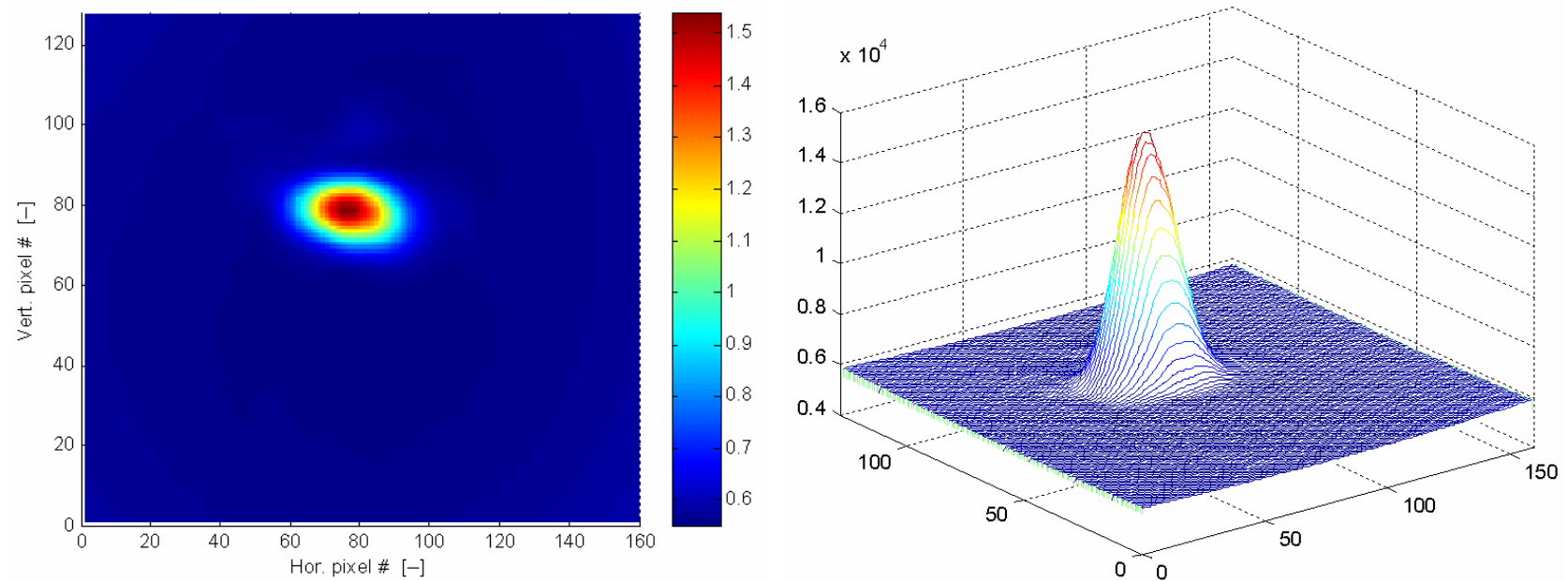

Figure 6 FFI of sample TAS-1F (23 cm, Gallium coated). Note that intensity distribution is elliptic.

For measuring the numeric aperture (NA) of the fiber, a horizontal and a vertical line crossing the maximum intensity in Figure 6 are selected and the pixel number are converted to the angle (Figure 7). The NA of the fiber can be calculated by the divergence angle $\alpha$. The relation is $\mathrm{NA}=\sin (\alpha / 2)$, where $\alpha$ is defined as the divergence angle at which the 
intensity is $5 \%$ of the maximum intensity. For the tested TAS fiber, the NA in the horizontal and vertical direction are found to be 0.12 and 0.06 respectively.
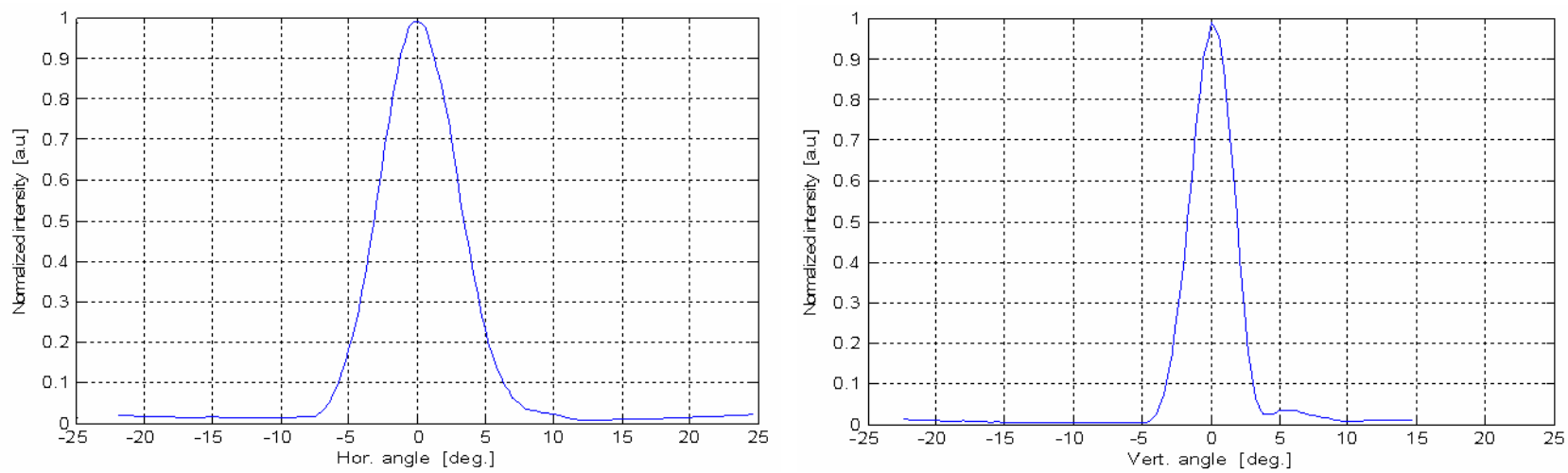

Figure 7 FFI in the horizontal and vertical direction of the main peak in Figure 6 (fiber sample TAS-1F, $23 \mathrm{~cm}$, Gallium coated). NA is 0.12 in the horizontal direction and 0.06 in the vertical direction.

\section{Circular intensity profile}

The elliptical intensity distribution observed in Figure 6 can most probably be attributed to the presence of non-rotationsymmetric mechanical stresses in the TAS-1 fiber introduced by winding the fiber on a drum during the manufacturing process. FFI tests performed later in the program on straight pieces (not winded on drum) of TAS-X (FTVM) and TAS3 (RTVM), showed a circular intensity distributions with a Gaussian profiles (figure 8).
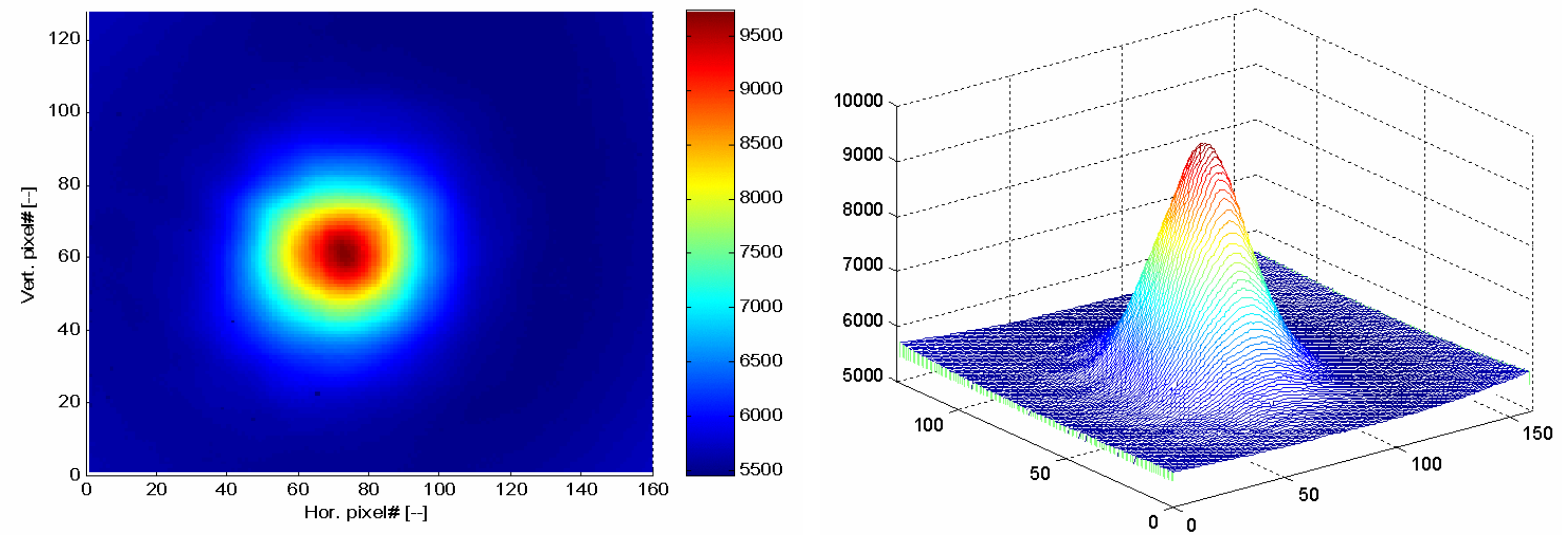

Figure 8 FFI of sample TAS-X showing a circular intensity distribution $(\mathrm{NA}=0.24)$.

\subsection{Attenuation measurement}

The attenuation of the single mode fibers is determined by the cut-back method. Within this program the measurements are performed at $10.6 \mu \mathrm{m}$ using the $\mathrm{CO}_{2}$ laser. The attenuation of the TAS- 1 and TAS-3 fiber was measured to be about $0.1 \mathrm{~dB} / \mathrm{cm}$ and $0.18 \mathrm{~dB} / \mathrm{cm}$ respectively. The higher attenuation of TAS-3 can be caused by the small NA found on this fiber. These values are comparable to the loss expected from the results of the bulk material testing.

\section{CONCLUSIONS}

The most stable glasses with transmission up to about $15 \mu \mathrm{m}$ are chalcogenide glasses. The chalcogenide glass type investigated and used in this project is based on the Te-As-Se (TAS) composition. The optical dispersion and the transmission window of different compositions of TAS glass are measured. A two-wavelength band system for 
DARWIN is presented. A number of TAS fibers for the short wavelength band are made by 3 different manufacturing methods. Far Field Intensity (FFI) distribution measurements are successfully carried out at $10.6 \mu \mathrm{m}$. For all three types of manufacturing methods applied, fiber samples have been characterized, showing a Gaussian distribution indicating single mode operation. The attenuation, measured by the cut-back method, is in the order of $0.1-0.18$ $\mathrm{dB} / \mathrm{cm}$.

\section{REFERENCES}

1. http://www.estec.esa.nl/spdwww/future/darwin/

2. D. Le Coq, Thesis, University of Rennes (June 25, 2002): Nouvelle Méthode d'Elaboration de Fibres Optiques Double Indice en Verre de Chalcogenures. Application a la Spectroscopie Infrarouge.

3. G. Fonteneau, C. Boussard-Pledel, D. Le Coq, Enceinte réactionelle pour la préparation de préformes pour fibres optiques, Demande de brevet, déposée en France le 21 juin 2002, sous le no. 0207732 